\title{
Rekabet Ortamında Akışkan Mal Satın Alma Süreci için Íki Seviyeli Matematiksel Model Önerisi
}

\author{
A Bi-Level Mathematical Model Proposal for Procurement Process of Liquid \\ Good in Competitive Environment
}

Gökhan ÖZÇELIK¹, Cevriye TEMEL GENCER²

\begin{abstract}
ÖZET
Literatürde zıt amaçları olan iki taraf arasındaki rekabetçi karar verme sürecinin modellenmesi "Şebeke Engelleme Problemi" olarak ifade edilmektedir. Bu çalışmada aynı tedarikçi kümesini kullanan rekabetçi iki firmanın akışkan mal satın alma süreci ele alınmıştır. Bu süreç, firmalardan birinin minimum maliyetle mal tedarik etmeye çalıştığı, diğerinin ise sahip olduğu engelleme bütçesi ile rakibinin minimum satın alma maliyetini en büyüklemeye çalıştığı bir rekabet ortamı için şebeke engelleme problemi şeklinde tasarlanmıştır. Bu problemdeki tedarik zinciri yapısı minimum maliyetli akış şebekesine dönüştürülerek, iki seviyeli (bi-level) matematiksel bir model önerilmiştir.
\end{abstract}

Anahtar kelimeler: Minimum maliyetli akış şebekesi, rekabetçi satın alma süreci, şebeke engelleme problemi.

\section{GíRiş}

Firmalar gün geçtikçe artan rekabet koşullarından zorlu şartlarla karşılaşabilmektedir. Bu amaçla firmalar üretim çevresinde ayakta kalabilmek adına pazarlama, satın alma gibi temel süreçlerinin yönetiminde sürekli yeni rekabetçi politikalar geliştirmek zorundadır. Bu kapsamda, aynı alanda faaliyet gösteren firmaların satın alma süreçlerini dikkate aldığımızda, firmalar arasında tedarikçilerdeki kapasite yüzünden rekabet doğabilmektedir. Diğer bir ifade ile bir firma belli bir tedarikçiden mal aldığı zaman tedarikçideki mal kapasitesini azaltacağından dolayı, diğer firmanın üretimini riske sokması muhtemeldir. Böylece firmalar rekabetçi bir mal satın alma süreci ile karşı karşıya kalacaktır.

Bu çalışmada, aynı alanda faaliyet gösteren rekabetçi iki firmanın (takipçi-lider) akışkan mal satın alma süreçleri analiz edilmiştir. İki firmanın da aynı tedarik zincirini kullandığı iki aşamalı tedarik zinciri

\begin{abstract}
Modeling of the competitive decision making process between two players which have opposite objectives is defined as "Network Interdiction Problem" in the literature. In this study, procurement process in which two competitive firms procure liquid good from the same set of the suppliers is handled. This process is designed as a network interdiction problem for competition environment in which one of the firms tries to procure goods with minimum cost, the other one tries to maximize the procurement cost of the competitor with owned interdiction budget, as well. In this problem transforming the structure of supply chain into minimum cost flow network, a bi-level mathematical model is proposed.
\end{abstract}

Keywords: Minimum cost flow network, competitive procurement process, network interdiction problem.

modeli ele alınmış, bu süreçte firmalardan birinin (liderin), diğerinin (takipçinin) minimum tedarik maliyetini en büyüklemesi hedeflenmiştir. Rekabet içindeki (zıt amaçları olan) iki firma ele alındığından dolayı model, şebeke engelleme problemi (ŞEP) şeklinde incelenmiştir.

ŞEP'te amaç birbirine zıt amaçları olan iki taraf arasındaki rekabetçi karar verme sürecini modellemektir.Şebekeüzerinde akışı en büyüklemeye çalışan, ya da hedefe en kısa yoldan ulaşmayı amaçlayan, kısaca şebekeden faydalanmaya çalışan şebeke kullanıcı ile bu akışı en küçüklemek, ya da en kısa yolu en büyüklemek amacıyla şebeke üzerinde engellemeler yapan yol kesici arasındaki oyundur. Bu oyun sırasında, takipçi sürekli olarak değişmiş şebekenin durumuna göre (engelleme durumlarına göre) en iyi (optimal) çözümü arayacaktır. Bahsedilen engelleme işlemi, kısıtlı engelleme bütçesiyle şebeke üzerindeki arkı yok eden ya da arkın uzunluğunun 
etkisini artıran (süresini, maliyetini) bir harekettir (Israeli ve Wood, 2002). Benzer şekilde bu engelleme işlemi problem yapısına göre düğümlerde, düğüm kapasitesini azaltarak da yapılabilir. Bu tip lider \& takipçi ilişkisi, statik Stackelberg (Stackelberg, 1952) oyununa benzemektedir.

Literatürde, ŞEP kapsamında karşılaştığımız ilk çalışmalara bakacak olursak; Wollmer $(1963,1964)$; Lubore vd. (1971); Ratliff vd. (1975); Malik vd. (1989) ve Ball vd. (1989) şebeke üzerinde en önemli ark (bağlantıyı) ya da arkları belirlemeye yönelikçalışmalar yapmıştır. Bu çalışmalarda temel olarak, "şebekeyi en kötü derecede sekteye uğratacak, hangi ark ya da arklar şebekeden kaldırılmalıdır?" sorusuna cevap aranmıştır. Corley ve David (1982); ise çalışmasında hem şebekedeki en önemli arkların hem de en önemli düğümlerin bulunmasına yer verirken, Corley ve Chang (1974) ve Nardelli vd. (2003) şebekedeki en önemli düğümün bulunmasına yönelik çalışmışlardır.

ŞEP'in sonraki yıllarda, şebekenin hassasiyetinin analizi ya da olası bir saldırıda şebekenin en az şekilde etkilenmesi için güçlendirilmesi gereken zayıf bileşenlerinin belirlenmesi gibi farklı alanlarda çalışıımıştır. Bununla birlikte ŞEP, terör saldırılarına karşı elektrik, su ya da haberleşme şebekelerinin savunulması problemleri ya da ilaç, uyuşturucu madde, nükleer madde kaçakçıı̆ı̆ında, terörist, suçlu ya da saldırganın kaçış olasııığının en küçüklenmesine yönelik sensör yerleşimi problemlerinde de ele alındığı görülmektedir. Ancak ŞEP'in temelini oluşturan iki çalışma alanından biri şebeke üzerinde maksimum akışın engellenerek en küçüklenmesi üzerinedir (Fulkerson ve Harding (1977); Golden (1978); Israeli ve Wood (2002), Khachiyan vd. (2007); Bayrak vd. (2008); Gutfraind vd. (2010); Romich vd. (2015); Borrero vd. (2016)). Diğer çalışma alanı ise en kısa yolun engellenerek en büyüklenmesi üzerinedir (Wollmer (1964); McMasters ve Mustin (1970); Wood (1993); Washburn ve Wood (1995); Cormican vd.
(1998); Lim ve Smith (2007); Royset ve Wood (2007); Akgün vd. (2011); Granata vd. (2013); Keshavarzi ve Fathabadi (2015); Janjarassuk ve Nakrachata-Amon (2016)). Bu çalışmaların birçoğu engelleme bütçesi dâhilinde ele alınmıştır. Diğer bir ifade ile engelleme işlemlerini kısıtlayan bir engelleme bütçesinin olduğu durumda engelleme planları elde edilmiştir (Bkz. Fulkerson ve Harding (1977); McMasters ve Mustin (1970); Wood (1993), Israeli ve Wood (2002), Cormican vd. (1998); Lim ve Smith (2007)).

Literatürde, tedarik zinciri üzerinde iki rekabetçi firmanın satın alma süreçlerini ŞEP şeklinde ele alan temel aynı yazarlar tarafından aynı yıl yapılmış iki çalışmanın olduğu görülmektedir: (Prince vd. (2013a, 2013b)). Bu çalışmalarda temel olarak tek aşamalı tedarik zinciri üzerinde, engelleme maliyetlerinin konkav olduğu durum dikkate alınmıştır. Modeller, ayrıştırma tekniklerine iyi uyan üç aşamalı karışık tam sayılı programlama ile formüle edilerek, modellerin çözümü için etkin bir kesme düzlemi algoritması geliştirmişlerdir. Bu çalışmada diğer çalışmalardan farklı olarak, kapasiteli ortak aktarma merkezlerinin varlığında Lim ve Smith (2007)'nin çalışması temel alınarak yeni bir problem yapısı geliştirilmiş ve model çözümü için Wood (1993)'un bu tip problemlerin çözümü için geliştirdiği dual alma tekniği kullanılmıştır.

\section{2. ÖNERILEN MATEMATIKSEL MODEL (MODL)}

MODL, Şekil 1'de gösterilen, kapasiteli yönlü arklar üzerinde $N$ düğüm seti ve $A$ ark seti olmak üzere $G=(N, A)$ şekilde tanımlanmıştır. İki aşamalı tedarik zinciri, kapasiteli tedarikçi düğümlerinden $(I \subset N)$, kapasiteli aktarma merkezlerinden $(J \subset N)$, firmalardan $(F \subset N)$, tedarikçiler ile aktarma merkezleri arasındaki arklardan $(i, j) \in A$ ve aktarma merkezleri ile firmalar arasındaki arklardan $(j, f) \in A$ oluşmaktadır.

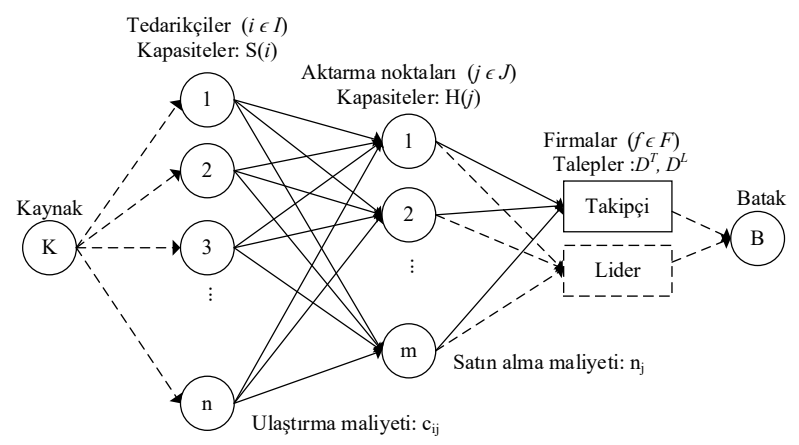

Şekil 1: Problemde Ele Alınan İki Aşamalı Tedarik Zinciri 
Şekil 1'degösterilentedarikzinciriyapısındakaynak düğümünden $(K)$ tedarikçi düğümlerine $(I \subset N)$ firmalardan ise batak düğüme $(B)$ olan akışlar ihmal edilmiştir. Bununla birlikte, her iki firmanın da aynı iki aşamalı tedarik zincirini kullandıkları, aktarma merkezlerinin kapasitelerinin her iki oyuncuya da yettiği, her iki oyuncunun da birbiri hakkında yeterli bilgiye sahip olduğu varsayılmaktadır. Ayrıca lider firmanın elindeki engelleme (anlaşmaya da kontrat) bütçesi dâhilinde tedarikçilerde antlaşma yapabildiği dolayısıyla takipçi firmayı engelleyebildiği (takipçi firmanın ilgili tedarikçiden mal satın almasının engellenebildiği) dikkate alınmıştır. Bu durumda satın alma prosedürü aşağıda tanımlandığı şekilde sıralı olarak gerçekleştirilmektedir;

(i) Öncelikle lider, engelleme bütçe dâhilinde engelleyeceği tedarikçilere karar verip, engelleme işlemini gerçekleştirecektir.

(ii) Daha sonra, takipçi, liderin engellediği tedarikçilerden arta kalan kapasiteyi kullanarak satın alma işlemini tamamlayacaktır.

MODL'de takipçi firma (follower) minimum toplam maliyet ile kendi talebini karşılamayı amaçlarken; lider firma (leader), tedarikçileri engelleyerek (düğüm kapasitesini sıfırlayarak) elindeki engelleme bütçesiyle takipçi firmanın başarabildiği minimum toplam maliyeti en büyüklemeyi amaçlamaktadır.

Akış, tedarikçilerden $i \in I, \quad(i=1,2, \ldots, n)$ aktarma merkezlerine $j \in J, \quad(j=1,2, \ldots, m)$ , aktarma merkezlerinden ise firmalara $(F \subset N)$ doğrudur. Tüm modeller, takipçi firmanın talebine yönelik kurulduğu için aslında $F$ setinde yalnızca bir eleman vardır $((f=1) ; f \in F)$. Dolayısıyla $S(F)=1$ 'dir. Bu amaçla, tüm modellerde $f$ indisi yerine, direkt olarak takipçinin talebini ifade eden $\left(D^{T}\right)$ parametresi kullanılmıştır. Bu durumda, tedarikçilerden, aktarma merkezlerine gerçekleşen akış miktarı $\left(x_{i j}\right)$ ve aktarma merkezlerinden firmaya (takipçiye) gerçekleşen akış $\left(y_{j}\right)$ takipçinin karar

$$
\begin{array}{lll}
z^{*}=\min _{x, y} \sum_{i \in I} \sum_{j \in J} c_{i j} x_{i j}+\sum_{j \in J} n_{j} y_{j} & \\
\sum_{j \in J} x_{i j} \leq S_{i} & \forall i \in I & : \alpha_{i} \\
\sum_{i \in I} x_{i j} \leq H_{j} & \forall j \in J & : \beta_{j} \\
y_{j}-\sum_{i \in I} x_{i j} \leq 0 & \forall j \in J & : \delta_{j}
\end{array}
$$




$$
\begin{array}{ll}
\sum_{j \in J} y_{j} \geq D^{T} & \\
x_{i j} \geq 0 & \forall i \in I, \forall j \in J \\
y_{j} \geq 0 & \forall j \in J
\end{array}
$$

Modelin amaç fonksiyonu Eş.1'de ifade edilmiştir. Eş.2 ve Eş.3'te ifade edilen kısıtlar, $i$. tedarikçiden satın alınan mal miktarını, sırasıyla, $i$. tedarikçinin ve $j$. aktarma merkezinin kapasiteleri ile sınırlandırmaktadır. Eş.4, akış denge kısıtlarını ifade etmektedir. Eş.5'te ifade edilen kısıt, toplam satın alınan mal miktarının takipçinin talebini

karşılamasını sağlamaktadır. Eş. 6 ve Eş.7, takipçinin karar değişkenleri için pozitiflik kısıtlarıdır. Modelde kısıtların yanında verilen $\alpha_{i}, \beta_{j}, \delta_{j}, \lambda$; kısıtlara ilişkin dual değişkenlerdir. Bu dual değişkenler bir sonraki bölümde içteki en küçükleme probleminin dualini alınırken kullanılacaktır.

\subsubsection{MOD-D: Takipçinin Minimum Maliyetli Mal Satın Alma Modelinin Duali}

$$
\begin{array}{lc}
z^{*}=\max _{\alpha, \beta, \delta, \lambda} \sum_{i \in I} S_{i} \alpha_{i}+\sum_{j \in J} H_{j} \beta_{j}+D^{T} \lambda \\
\alpha_{i}+\beta_{j}-\delta_{j} \leq c_{i j} & \forall i \in I, \forall j \in J \\
\delta_{j}+\lambda \leq n_{j} & \forall j \in J \\
\alpha_{i} \leq 0 & \forall i \in I \\
\lambda \geq 0 & \forall j \in J \\
\beta_{j} \leq 0 ; \delta_{j} \leq 0 &
\end{array}
$$

\subsection{Lider Firmanın Probleminin Formülasyonu}

Bu bölümde lider firmanın problemi iki seviyeli max-min yapısında modellenmiş daha sonra doğrusal olmayan tek seviyeli karışık tam sayılı modele çevrilmiştir. İki seviyeli modelde, sabit $w_{i}$ karar değişkeni için, içteki en küçükleme modeli takipçinin tedarik modelidir. En büyükleme problemi ise liderin problemidir ve liderin karar değişkeni $w_{i} ; i \in I$ düğümü (tedarikçiler) engellenirse 1 , diğer durumlarda 0 değerini alacaktır. Liderin amacı, $i \in I$ düğümlerini engelleyerek, takipçinin minimum tedarik maliyetini engelleme sayısına bağlı olarak en büyüklemektir.

\subsubsection{MODL-BI: İki seviyeli MODL}

MODL-BI, MOD'da takipçi firmanın tedarik ettiği akışkan malların minimum toplam maliyetini engelleme bütçesine bağlı olarak en büyüklemeye çalışmaktadır.

$$
\begin{aligned}
& z^{*}=\max _{w \in W} \min _{x, y} \sum_{i \in I} \sum_{j \in J} c_{i j} x_{i j}+\sum_{j \in J} n_{j} y_{j} \\
& \sum_{j \in J} x_{i j} \leq S_{i}\left(1-w_{i}\right) \quad \forall i \in I \quad: \phi_{i} \\
& \sum_{i \in I} x_{i j} \leq H_{j} \quad \forall j \in J \quad: \beta_{j} \\
& y_{j}-\sum_{i \in I} x_{i j} \leq 0 \quad \forall j \in J \quad: \delta_{j}
\end{aligned}
$$


$\sum_{j \in J} y_{j} \geq D^{T}$

$x_{i j} \geq 0$

$\forall i \in I, \forall j \in J$

$y_{j} \geq 0$

$\forall j \in J$
$: \lambda$

MODL-BI'de, amaç fonksiyonu Eş.14'te ifade edilmiştir. Eş. 15 ve Eş.16 'da ifade edilen kısıtlar, $i$. tedarikçiden satın alınan mal miktarını, sırasıyla, $i$. tedarikçinin ve $j$. aktarma merkezinin kapasiteleri ile sınırlandırmaktadır. Eş. $15^{\prime}$ te, $w_{i}=1$ ise, bu lider firmanın $i$. tedarikçiyi engellediğini göstermektedir. $\mathrm{Bu}$ durumda engellenen tedarikçinin kapasitesi sıfırlanacaktır, dolayısıyla takipçi firma bu tedarikçiden mal satın alamayacaktır. Benzer şekilde, $w_{i}=0$ olursa, lider firmanın $i$. tedarikçiyi engellemediği, yani takipçi firma için, $i$. tedarikçinin kapasitesinde herhangi bir değişiklik olmadığı anlamına gelmektedir. Eş. 17, akış denge kısıtlarını ifade etmektedir. Eş. 18'te ifade edilen kısıt, toplam satın alınan mal miktarının takipçinin talebini karşılamasını sağlamaktadır. Eş. 19 ve Eş. 20, takipçinin karar değişkenleri için pozitiflik kısıtlarıdır.

Eş.21'deki kısıtlar ise belli bir engelleme bütçesi $(R)$ dâhilinde lider firmanın engelleyebileceği tedarikçi sayısını sınırlandırmaktadır. Burada engelleme değişkeni $\left(w_{i}\right)$ 0-1 (binary) karar değişkeni, $\left(r_{i}\right)$ ise $i$ tedarikçisini engellemek için gerekli maliyeti gösteren parametredir. Model çözümü için; $w$ karar değişkeni sabit tutularak içteki en küçükleme probleminin duali alınmıştır ve daha sonra $w$ karar değişkeni serbest bırakılmıştır. Böylece, lider firma için karışık tam sayılı doğrusal olmayan max-max modeli elde edilmiştir. Bir sonraki bölümde, doğrusal olmayan bu model doğrusallaştırıımıştır.

\subsubsection{MODL: Lider firmanın karışık tam sayılı doğurusal olmayan modeli ve modelin doğrusallaştırılması}

Wood (1993) çalışmasında problem yapısı uni-modular olduğunda, liderin karar değişkenin sabit tutularak iki seviyeli maksimum akış şebeke engelleme modelinin tek seviyeli karışık tam sayılı modele dönüştürülebileceğini ispatlamıştır. Bu tezde de, problem yapısı uni-modular olduğu için modelin tek seviyeye indirgenmesinde bu dönüşüm kullanılmıştır.

Dolayısıyla, $w_{i}$ karar değişkeninin geçici olarak sabit tutulup içteki en küçükleme modelinin duali alınarak ve daha sonra $w_{i}$ karar değişkeni serbest bırakılarak, model tek seviyeli (max-max) karışık tam sayılı doğrusal olmayan matematiksel modele dönüştürülmüştür. Böylece, iki seviyeli model, aynı amaç fonksiyonu yapısına (max) sahip, ancak değişkenlerin farklı setleri üzerinde (liderin primal değişkeni $\left(w_{i}\right)$; takipçinin dual değişkenleri $\left.\left(\phi_{i}, \beta_{j}, \delta_{j}, \lambda\right)\right)$ tek seviyeli modele indirgenmiştir.

$$
\begin{array}{ll}
z^{*}=\max _{w} \max _{\phi, \beta, \delta, \lambda} \sum_{i \in I} S_{i}\left(1-w_{i}\right) \phi_{i}+\sum_{j \in J} H_{j} \beta_{j}+D^{T} \lambda \\
\phi_{i}+\beta_{j}-\delta_{j} \leq c_{i j} & \forall i \in I, \forall j \in J \\
\delta_{j}+\lambda \leq n_{j} & \forall j \in J \\
\sum_{i \in I} r_{i} w_{i} \leq R & \\
\phi_{i} \leq 0 & \forall i \in I \\
\lambda \geq 0 & \forall j \in J \\
\beta_{j} \leq 0 ; \delta_{j} \leq 0 & \forall i \in I \\
w_{i} \in\{0,1\} &
\end{array}
$$


Görüldüğü üzere MODL'deki $\phi_{i}$, MOD-D'deki $\alpha_{i}^{\prime}$ 'nın rolünü üstlenmiştir. Bununla birlikte $\phi_{i} \leq 0$ ve $w_{i} \in\{0,1\}$ olduğu bilinmektedir. Dolayısıyla, model eniyilemede herhangi bir kayıp olmaksızın doğrusallaştırılabilir. Bu amaçla $\phi_{i}\left(1-w_{i}\right)$ yerine, $\mu_{i} \leq 0$ değişkeni yazılımış ve modele $\mu_{i} \leq \phi_{i}+w_{i} M$ kısıt kümesi (33) ilave edilmiştir ( $M$ yeterince büyük bir sayı). Eğer en iyi çözümde $w_{i}=1$ olursa, $\phi_{i} S_{i}\left(1-w_{i}\right)$ ifadesi 0 'a; $w_{i}=0$ olursa $\phi_{i} S_{i}$ 'a eşit olacaktır. Yapılan değişime göre $\phi_{i} S_{i}\left(1-w_{i}\right)$ ifadesi yerine artık $S_{i} \mu_{i}$ yazılabilir. Yapılan doğrusallaştırmaya göre, en iyi çözümde $w_{\mathrm{i}}=1$ olursa, $\mu=0$ olmalıdır. En büyükleme problemi için Eş. 33 ve 39'da tanımlanan kısıtlar bu durumu sağlamaktadır. (33). kısıt yeterince büyük sağ taraf değerine sahip olduğunda ve (33) ve (39) kısıtları eş zamanlı düşünüldüğünde $\mu_{i}=0$ olacaktır. Aynı şekilde, eğer en iyi çözümde, $w_{i}=0$ olursa $\mu_{i}=\phi_{i}$ olacaktır. Bu durumda da en büyükleme problemi için (33) ve (39). kısıtlar sağlanacaktır. (33) ve (39). kısıtlar eş zamanlı düşünüldüğünde $\mu_{i}=\phi_{i}$ eşitliği doğrulacaktır. Bu yargılar, doğrusallaştırmanın geçerliliğini kanıtlamaktadır.

\subsubsection{MODL-S: MODL'nin tam formülasyonunun son hali}

$$
\begin{array}{ll}
z^{*}=\max _{w, \phi, \beta, \delta, \lambda, \mu} \sum_{i \in I} S_{i} \mu_{i}+\sum_{j \in J} H_{j} \beta_{j}+D^{T} \lambda \\
\phi_{i}+\beta_{j}-\delta_{j} \leq c_{i j} & \forall i \in I, \forall j \in J \\
\delta_{j}+\lambda \leq n_{j} & \forall j \in J \\
\mu_{i} \leq \phi_{i}+M w_{i} & \forall i \in I \\
\sum_{i \in I} r_{i} w_{i} \leq R & \forall i \in I \\
\phi_{i} \leq 0 & \\
\lambda \geq 0 & \forall j \in J \\
\beta_{j} \leq 0 ; \delta_{j} \leq 0 & \forall i \in I \\
\mu_{i} \leq 0 & \forall i \in I \\
w_{i} \in\{0,1\} &
\end{array}
$$

(Not: *M yeterince büyük bir sayl)

MODL-S ile lider firma hem engelleme planını elde etmiş hem de engellemeden sonra takipçi firmanın minimum toplam maliyetini belirlemiş olacaktır. Burada, lider firma engelleme sonrası, uygun maliyetli tedarikçilerle anlaşma yaptığı için doğal olarak anlaşma yaptığı tedarikçilere yönelecektir. Dolayısıyla, ilk duruma göre maliyetini düşürmüş olmakla birlikte; engelleme bütçesine göre kar zarar durumu değişkenlik gösterebilecektir. Bu durumda, karar verici (lider firma) amacına en uygun politikayı belirlemek zorundadır. Eğer amacı yalnızca takipçi firmayı zarara uğratmak ise engelleme bütçesini oldukça fazla tutmalıdır. Karşı tarafı zarara uğratıp aynı zamanda da kar etmek istiyorsa, ortalama bir engelleme bütçesi ile bu amacına ulaşabilir. Bunun için de engelleme bütçesinin ne olması gerektiği konusunda ciddi bir analiz yapılması gerekmektedir.

\section{UYGULAMA}

Yapılan uygulamada, iki rakip firmanın aynı 6 tedarikçiden mal aldığı ve ortak kullanılan aktarma merkezlerinin bulunduğu bir problem tasarlanmıştır. Illk olarak lider firmanın takipçi firmanın önüne geçmek adına belli tedarikçilerin kapasitesini engellediği (belli bir bütçe karşıı̆ı ilgili tedarikçilerle anlaşma yaptığı) daha sonra kalan kapasiteden takipçi firmanın mal tedarik ettiği probleme ilişkin bilgiler Tablo 1'de verilmiştir. 
Tablo 1: Probleme İlişkin Veriler

\begin{tabular}{|c|c|c|c|c|c|c|c|c|}
\hline & \multicolumn{2}{|c|}{ Kapasiteler (galon) } & \multicolumn{6}{|c|}{ Maliyetler (Pb/br) } \\
\hline & $\begin{array}{l}\text { Tedarikçiler } \\
\left(U_{i}\right)\end{array}$ & $\begin{array}{l}\text { Aktarma Merkez- } \\
\text { leri }\left(H_{j}\right)\end{array}$ & Ulaşt & na $\left(c_{i j}\right)$ & & & $\begin{array}{l}\text { Satın Alma } \\
\left(n_{j k}\right)\end{array}$ & $\begin{array}{l}\text { Engelleme } \\
\text { (Anlaşma) } \\
\text { Maliyeti }\left(r_{i}\right)\end{array}$ \\
\hline 1. & 120 & a bölgesi (200) & $c_{1 a}=2$ & $c_{2 c}=1$ & $c_{4 a}=3$ & $c_{5 c}=2$ & $\mathrm{n}_{\mathrm{ak}}=2,80$ & 20 \\
\hline 2. & 116 & b bölgesi (150) & $c_{1 b}=8$ & $c_{2 d}=5$ & $c_{4 b}=2$ & $c_{5 d}=3$ & $\mathrm{n}_{\mathrm{bk}}=1,86$ & 22 \\
\hline 3. & 150 & c bölgesi (200) & $c_{1 c}=3$ & $c_{3 a}=5$ & $c_{4 c}=7$ & $c_{6 \mathrm{a}}=5$ & $\mathrm{n}_{\mathrm{ck}}=2,75$ & 21 \\
\hline 4. & 145 & d bölgesi (200) & $c_{1 d}=1$ & $c_{3 b}=3$ & $c_{4 d}=8$ & $c_{6 b}=4$ & $\mathrm{n}_{\mathrm{dk}}=1,98$ & 25 \\
\hline 5. & 100 & & $c_{2 a}=2$ & $c_{3 c}=4$ & $c_{5 a}=4$ & $c_{6 c}=4$ & & 24 \\
\hline 6. & 98 & & $c_{2 b}=1$ & $c_{3 d}=5$ & $c_{5 b}=4$ & $c_{6 d}=4$ & & 20 \\
\hline
\end{tabular}

(Not: Talep iki firma için de DL, DT: 350br, Lider firmanın engelleme bütçesi $(R \leq 50)$ )

Hiç bir engelleme olmadığında, takipçi firma 350 br'lik talebini 1,2 ve 4 . tedarikçileri ve b,c ve $d$ bölgesindeki aktarma merkezlerini kullanarak 1200,6 pb'ne (MOD ya da MOD-D ile bulunabilir) karşılayabilmektedir. Bu durumda lider firmada, kalan kapasiteden mal ihtiyacını $3,4,5$ ve 6 . tedarikçileri ve $a, c$ ve $d$ bölgesindeki aktarma merkezlerini kullanarak 2196,4 pb'ne karşılamaktadır (MOD ya da MODD'de takipçi firmanın mal alımı sonrası kapasiteler güncellenerek bulunabilir).

Engelleme bütçesi $50 \mathrm{pb}$ olduğunda, bu kez takipçi firma talebini $3,4,5$ ve 6 . tedarikçileri ve $b, c$ ve $d$ bölgesindeki aktarma merkezlerini kullanarak $1658,54 \mathrm{pb}$ 'ne karşılayacak (MODL-S ile bulunur), lider firma ise engellediği (anlaşma yaptığı 1. ve 2.) tedarikçilere yönelerek talebini $1733.46 \mathrm{pb}$ 'ne 1.2 . ve 3. tedarikçileri ve $a, c$ ve $d$ bölgesindeki aktarma merkezlerini kullanarak karşılayacaktır (MOD ya da MOD-D'de takipçi firmanın mal alımı sonrası kapasiteler güncellenerek bulunabilir).

Sonuç olarak, engelleme bütçesi dâhilinde lider firma takipçi firmayı 457,94pb zarara uğratırken, kendisi de mal satın alma açısından 462,94pb kara geçmiştir. Ancak 1 ve 2 . tedarikçileri engellediği için engelleme bütçesinin $42 \mathrm{pb}$ 'ni kullanmıştır. Bu maliyeti de hesaba katıldığında lider firma toplamda

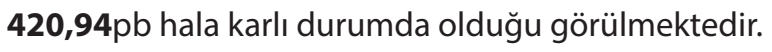

\section{SONUÇ}

Firmalar rekabet ortamında ayakta kalabilmek adına satın alma, üretim, pazarlama gibi süreçler için çeşitli politikalar geliştirmektedir. Çalışmada, firmaların rekabet ortamındaki satın alma süreçleri Şebeke Engelleme Problemi şeklinde ele alınmıştır. Bu kapsamda, satın alma sürecinde, bir firmanın (lider) rakip firmayı (takipçi) belli bir engelleme bütçesi dâhilinde maliyetler açısından alt edebileceği iki seviyeli karışık tam sayılı matematiksel model oluşturulmuş, daha sonra bu model tek seviyeye indirgenerek doğrusal karışık tam sayılı modele dönüştürülmüştür. Önerilen nihai modele göre $(M O D L-S)$, lider firmanın engelleme bütçesi kapsamında hangi tedarikçileri engellemesi gerektiği ve bu durumda rakip firmanın toplam maliyetinin ne olacağı sorularına cevap bulunmuştur. Lider firma tarafından belirlenen engelleme bütçesinin miktarı lider firmanın engellemeden dolayı ortaya çıkan toplam maliyetini (satın alma maliyeti + engelleme maliyeti) etkilemektedir. Düşük belirlenmesi firmayı kar durumunda tutabilecekken; yüksek belirlenmesi zarar ettirebilir. Bu durumda engelleme bütçesinin ne olması gerektiği karar vericiye bağlıdır. Engelleme ya da tedarikçilerle anlaşma durumu riskli bir süreçtir, tedarikçileri ikna edememe ya da yüksek fiyatlara ikna etme durumları oluşabilir. Illeriki çalışmalarda, engelleme durumunda engellemeden dolayı karşılaşılabilecek risk yapıları ya da risk maliyetleri de modele ilave edilerek çok amaçlı modeller oluşturulabilir. 


\section{KAYNAKÇA}

Akgün, İ., Tansel, B.Ç. ve Wood, R.K. (2011) “The Multi-Terminal Maximum-Flow Network-Interdiction Problem" European Journal of Operational Research, 211(2), 241-251.

Ball, M.O., Golden, B.L. ve Vohra, R.V. (1989) "Finding the Most Vital Arcs in a Network" Operations Research Letters, 8(2), 73-76.

Bayrak, H. ve Matthew D. Bailey, M.D. (2008) "Shortest Path Network Interdiction with Asymmetric Information" Networks, 52(3), 133-140.

Borrero, J.S., Prokopyev, O.A. ve Sauré, D. (2016) "Sequential Shortest Path Interdiction with Incomplete Information" Decision Analysis, 13(1), 68-98.

Corley, H. ve Chang, H. (1974) "Finding the n Most Vital Nodes in a Flow Network" Manage Science, 21, 362-364.

Corley, H. ve David, Y.S. (1982) "Most Vital Links and Nodes in Weighted Networks" Operations Research Letters, 1(4), 157-160.

Cormican, K.J., Morton, D.P. ve Wood, R.K. (1998) "Stochastic Network Interdiction" Operations Research, 46(2), 184-197.

Fulkerson, D.R. ve Harding, G.C. (1977) "Maximizing the Minimum Source-Sink Path Subject to a Budget Constraint" Mathematical Programming, 13(1), 116-118.

Golden, B. (1978) "A Problem in Network Interdiction” Naval Research Logistics Quarterly, 25(4), 711-713.

Granata, D., Steeger, G. ve Rebennack, S. (2013) "Network Interdiction via a Critical Disruption Path: Branch-and-Price Algorithms" Computers \& Operations Research, 40(11), 2689-2702.

Gutfraind, A., Hagberg, A.A., Izraelevitz, D. ve Pan, F. (2010) "Interdiction of a Markovian Evader" Paper presented at the INFORMS Computing Society Conference.

Israeli, E. ve Wood, R.K. (2002) "Shortest-Path Network Interdiction" Networks, 40(2), 97-111.

Janjarassuk, U. ve Nakrachata-Amon, T. (2016) “ $A$ Simulated Annealing Algorithm to the Stochastic Network Interdiction Problem" Paper presented at the IEEE International Conference on Industrial Engineering and Engineering Management.

Keshavarzi, R. ve Fathabadi, H.S. (2015) "Multi-Commodity Multi-Source-Sinks Network Flow Interdiction Problem with Several Interdictors" Journal of Engineering and Applied Sciences, 10(6), 118-122.

Khachiyan, L., Boros, E., Borys, K., Elbassioni, K., Gurvich, V., Rudolf, G. ve Zhao, J. (2007) "On Short Paths Interdiction Problems: Total and Node-Wise Lim- ited Interdiction" Theory of Computing Systems, 43(2), 204-233.

Lim, C. ve Smith, J.C. (2007) "Algorithms for Discrete And Continuous Multicommodity Flow Network Interdiction Problems" IIE Transactions, 39(1), 15-26.

Lubore, S., Ratliff, H. ve Sicilia, G.T. (1971) "Determining the Most Vital Link in a Flow Network" Naval Research Logistics Quarterly, 18(4), 497-502.

Malik, K., Mittal, A.K. ve Gupta, S.K. (1989) “The k Most Vital Arcs in the Shortest Path Problem" Operations Research Letters, 8(4), 223-227.

McMasters, A.W. ve Mustin, T.M. (1970) "Optimal Interdiction of a Supply Network" Naval Research Logistics Quarterly, 17(3), 261-268.

Nardelli, E., Proietti, G. ve Widmayer, P. (2003) "Finding the Most Vital Node of a Shortest Path" Theoretical Computer Science, 296(1), 167-177.

Prince, M., Smith, J.C. ve Geunes, J. (2013a). "A Three-Stage Procurement Optimization Problem under Uncertainty" Naval Research Logistics (NRL), 60(5), 395412.

Prince, M., Geunes, J. ve Smith, J.C. (2013b) "Procurement Allocation Planning with Multiple Suppliers under Competition" International Journal of Production Research, 51(23-24), 6900-6922.

Ratliff, H.D., Sicilia, G.T. ve Lubore, S.H. (1975) "Finding the Most Vital Links in Flow Networks" Management Science, 21(5), 531-539.

Royset, J.O. ve Wood, R.K. (2007) "Solving the Bi-Objective Maximum-Flow Network-Interdiction Problem" INFORMS Journal on Computing, 19(2), 175184.

Stackelberg, v.H. (1952) "The Theory of the Market Economy" London, UK: William Hodge \& Co.

Washburn, A. ve Wood, K. (1995) "Two-Person Zero-Sum Games for Network Interdiction" Operations Research, 43(2), 243-251.

Wollmer, R. (1963) "Some Methods for Determining the Most Vital Link in a Railway Network" Santa Monica, California: Rand Corporation.

Wollmer, R. (1964) "Removing Arcs from a Network" Operations Research, 12(6), 934-940.

Wood, R.K. (1993) "Deterministic Network Interdiction" Mathematical and Computer Modelling, 17(2), 1-18.

Yates, J. ve Sanjeevi, S. (2013) "A Length-Based, Multiple-Resource Formulation for Shortest Path Network Interdiction Problems in the Transportation Sector" International Journal of Critical Infrastructure Protection, 6(2), 107-119. 